missing data, a mixed effects model was used using residual maximum likelihood. Clinic visit and disease severity were included as factors. Utility values were assumed to be missing at random.

Results The estimated utility of COPD patients according to levels of disease severity was as follows: Mild $=0.820$ (95\% CI: 0.800 0.840 ); Moderate $=0.801$ (95\%CI: 0.794-0.809); Severe $=0.774$ (95\% CI: 0.767-0.782); and Very Severe $=0.743$ (95\% CI: 0.730 0.756). The correlation between increasing disease severity and decreasing patients' utility demonstrated the internal validity of the data.

Conclusion This analysis provides estimates of utility by COPD disease severity based on one of the largest sample sizes used to date, which is essential for cost-utility analyses that help inform healthcare decisions.

1. Kind P et al. "Variations in population health status: results from a United Kingdom national questionnaire survey." BMJ 316, no. 7133 (Mar 1998): 736-41.

\section{P85 ASSESSING THE PATIENT EXPERIENCE OF COPD CARE USING AN IPSOS MORI QUESTIONNAIRE AS A PATIENT REPORTED EXPERIENCE MEASURE (PREM)}

doi:10.1136/thoraxjnl-2012-202678.327

'DJ Powrie, 'M Ali, 'S Ansari, 'KG Lingam, ${ }^{2} \mathrm{P}$ Mulley, ${ }^{3}$ S Hope, 'AG Davison. 'Southend University Hospital, Westcliff on Sea, UK; ${ }^{2}$ GlaxoSmithKline, Stockley Park West, UK; ${ }^{3} /$ psos MORI, London, UK

Patient reported experience measures provide vital information on the experience that patients have regarding their care and thus have become a recent focus of interest. We have developed a questionnaire to assess PREMS in patients with COPD attending a hospital outpatient clinic. The purpose of the questionnaire was to assess whether the processes that are necessary to improve outcomes in COPD had been dealt with in the clinic and understood by the patient. Also to assess in a similar way the understanding of COPD by the patient and whether they were satisfied with the consultation.

Results The questionnaire was completed by 142 new and follow up patients. Results are presented in the table below. $92 \%$ of patients had spirometry performed at their visit and $78 \%$ had the results explained. Before the consultation $57 \%$ of patients thought they had a good understanding, 30\% moderate and $13 \%$ little or no understanding of their condition. Afterwards these improved to $67 \%, 23 \%$ and $10 \%$ respectively. $29 \%$ believed that their knowledge of how to reduce exacerbations had increased after the consultation. $87 \%$ of patients thought that the length of the review was about right (average 20 minutes). $85 \%$ were satisfied overall with the consultation.

Conclusion We have studied COPD care from the patient's perspective. We have found evidence that simple clinician-led measures (such as flu vaccination and spirometry) have been explained either in clinic or previously and understood by the patient. We were less successful in dealing with more complex patient-centred experiences such as understanding of the disease and self management of exacerbations. Following this study we plan to develop a questionnaire dealing with the patient's understanding of their disease that would

Abstract P85 Table 1

\begin{tabular}{lccc}
\hline During your consultation were you told? & $\begin{array}{c}\text { Explained in } \\
\text { clinic (\%) }\end{array}$ & $\begin{array}{c}\text { Explained } \\
\text { before (\%) }\end{array}$ & total \\
\hline How to access flu vaccination & 65 & 22 & 87 \\
How to access pneumonia vaccination & 46 & 25 & 71 \\
How to use your inhalers & 59 & 33 & 92 \\
How medicines would benefit you & 56 & 27 & 83 \\
How to access to smoking cessation & 69 & 17 & 86 \\
How COPD may affect you & 43 & 35 & 78 \\
What to do if symptoms get worse & 20 & 46 & 66 \\
\hline
\end{tabular}

be filled in before attending clinic. This would allow areas of concern to the patient to be specifically addressed in the consultation. In addition the questionnaire could be used to provide feedback to individual clinicians on their effectiveness in outpatient clinics.

\section{Cystic Fibrosis: diagnosis to therapy}

\section{P86 THE SCREENING AND DIAGNOSIS OF CYSTIC FIBROSIS- RELATED DIABETES IN THE UNITED KINGDOM}

doi:10.1136/thoraxjnl-2012-202678.328

${ }^{1} \mathrm{KL}$ Wickens-Mitchell, ${ }^{2} \mathrm{FJ}$ Gilchrist, ${ }^{1} \mathrm{~W}$ Lenney. ${ }^{1}$ Keele University, Keele, UK; ${ }^{2}$ University Hospital of North Stafforshire, Stoke on Trent, UK

Introduction Cystic fibrosis related diabetes (CFRD) affects 17\% of CF patients in the UK and is increasing in prevalence. It has a major detrimental impact on pulmonary function, nutritional status and survival; these effects are frequently seen prior to diagnosis. The UK CF Trust guidelines regarding CFRD screening, diagnosis and management differ from those used in Europe and the USA. We conducted a study to establish current UK clinical practise.

Methods A questionnaire was emailed to consultants at each of the 48 UK CF specialist centres. Data were gathered on the screening and diagnosis of CFRD as well as the personnel involved.

Results Completed questionnaires were returned by 39/48 centres (81\%). Only 3/21 (14\%) paediatric centres begin screening at 12 years (as per the UK CF Trust guidelines), with the majority; 11/21 (52\%) starting to screen children at 10 years (as per the European and USA guidelines). Five centres start screening at a child's first annual review. The most common test used to screen patients for CFRD is the oral glucose tolerance test (OGTT) which is used in 33/39 (85\%) centres. However, this tool is only used in isolation by $3 / 33(9 \%)$ centres. More commonly, results of the OGTT are combined with random blood glucose tests and/or $\mathrm{HbA}_{1 \mathrm{C}}$ measurement. The test most frequently used to diagnose CFRD is home blood glucose monitoring which is undertaken in $32 / 39$ centres (82\%). Again this is rarely used in isolation, more commonly combined with $\mathrm{HbA}_{1 \mathrm{c}}$ and/ or with the results of a continuous glucose monitoring system (CGMS). CGMS is undertaken for diagnosis in 23/39 centres (59\%). The decision to initiate insulin therapy was most often shared between a CF consultant and diabetologist. However, in 4/14 (36\%) centres a diabetic nurse specialist had sole responsibility.

Conclusions In UK clinical practise the screening and diagnosis of CFRD is not uniform. Various methods are used and there is poor adherence to UK CF Trust guidelines. However, these guidelines from 2004 are somewhat out-dated and need to be updated to reflect the current best available evidence. This is likely to decrease the variation in practise.

\section{P87 THE IMPACT OF RESPIRATORY VIRUSES AND PULMONARY EXACERBATIONS ON FEV1 DECLINE IN ADULTS WITH CYSTIC FIBROSIS}

doi:10.1136/thoraxjnl-2012-202678.329

'WG Flight, ${ }^{2}$ KJ Mutton, 'AK Webb, 'RJ Bright-Thomas, 'AM Jones. 'Manchester Adult Cystic Fibrosis Centre, Manchester, United Kingdom; '2Dept of Virology, Manchester Royal Infirmary, Manchester, United Kingdom

Introduction Viral respiratory infection (VRI) is associated with an increased rate of decline in lung function in children with cystic fibrosis (CF) but the long-term clinical impact of VRI in adults is poorly described. We performed a prospective observational study to determine the effect of VRI on lung function in adults with CF. Methods 100 adults with CF were followed for 12 months. Patients were seen every two months routinely and also at onset of new respiratory symptoms. Sputum, nose- and throat-swabs were 